\title{
Preparation and Characterization of Blending Sulfonated Poly(ether ether ketone) with Charged Surface Modifying Macromolecules
}

\author{
M. N. A. Mohd-Norddin ${ }^{1}$, A. F. Ismail ${ }^{2,}$, T. Matsuura ${ }^{3}$ \& D. Rana ${ }^{4}$ \\ ${ }^{1 \& 2}$ Membrane Research Unit, Gas Engineering Department, Faculty of Chemical and Natural Resources \\ Engineering, Universiti Teknologi Malaysia, 81310 Skudai, Johor, Malaysia \\ ${ }^{3 * 4}$ Industrial Membrane Research Institute, Department of Chemical and Biological Engineering, University of \\ Ottawa, 161 Louis Pasteur St, Ottawa, ON, KIN 6N5, Canada
}

\begin{abstract}
Charged surface modifying macromolecule (CSMM) end capped with sulfonic group was prepared and blended into sulfonated poly (ether ether ketone) (SPEEK). The modified membrane was characterized for polymer electrolyte membrane application; i.e. a SPEEK/CSMM blend membrane was compared to a SPEEK membrane and a Nafion 112 membrane for the thermal and mechanical stability, hydrophilicity and water uptake. Thermal and mechanical stability of the blend membrane were slightly reduced from that of the SPEEK membrane but still higher than that of the Nafion 112 membrane. The cSMM which was end-capped with sulfonic group has increased the total $-\mathrm{SO}_{3} \mathrm{H}$ group in the blend membrane. As a result, the blend membrane showed an increase in hydrophilicity and water uptake as compared to the pristine membrane. The addition of $\mathrm{CSMM}$ with an additional $-\mathrm{SO}_{3}{ }^{-}$charge has enabled the water uptake to be increased which is crucial to enhance the proton conductivity that of SPEEK. Moreover, the extra $-\mathrm{SO}_{3}{ }^{-}$can facilitate the transfer of proton better. This study found that cSMM is a good candidate as an additive for SPEEK in improving its function as polymer electrolyte membrane.
\end{abstract}

Keywords: Direct methanol fuel cell, proton exchange membrane, sulfonated poly(ether ether ketone), charged surface modifying macromolecule, blend membrane

\subsection{INTRODUCTION}

The heart of direct methanol fuel cell (DMFC) is the proton exchange membrane (PEM) which separates catalyzed cathode and anode electrodes. The conventional PEM material used in DMFC is Nafion ${ }^{\circledR}$. However, it has three major disadvantages of high cost, loss of conductivity at temperatures above $80^{\circ} \mathrm{C}$ and high methanol crossover that has limited its applications. The high methanol crossover from anode to cathode is a major problem since it wastes fuel and causes performances losses at the cathode due to the

\footnotetext{
* Correspondence to: A. F. Fauzi (email:afauzi@utm.my)
}

consumption of oxygen and leads to poisoning of the catalyst.

Various efforts were made along different directions aimed to develop alternative replacement of Nafion which are more economical, have higher operating temperatures, as well as higher proton conductivity and low methanol permeability. Among them, particularly poly(ether ether ketone) (PEEK) has been extensively studied by many researchers for instance on crosslinking [1], composite membranes with inorganic materials $[2,3]$, blend membranes with other polymers [4] and surface modification $[5,6]$ such as plasma treatment and grafting reaction, surface coating or combining the said methods. PEEK must be sulfonated in order to be used as PEM 
material. Sulfonation is a well known process to increase the hydrophilicity and proton conductivity of polymer by attaching sulfonic groups to the polymers' chain. The attached sulfonic groups can offer and retain relatively higher water due to the enhanced antifouling capacity and favorable hydrodynamic environment of the membrane [7], which is also a very important mechanism for proton conducting.

In the current study, a modification of sulfonated PEEK (SPEEK) by blending surface modifying macromolecules (SMM) method is adopted. SMM is a simple blending method which uses the concept of surface segregation in polymer science as detailed elsewhere [8-11]. SMM when introduced as an additive in a base polymer will migrate to the surface and change the chemistry of the surface while maintaining its bulk properties. The migration of the hydrophilic or hydrophobic SMM segment is according to the thermodynamic principles of the tendency to minimize the interfacial energy. Due to this fact, when a membrane of blended polymer with SMM is cast, the polymer with the lowest surface energy of hydrophilic or hydrophobic will migrate and concentrate at the top or air interface. The bulk properties remain almost unchanged for a very small amount of SMM, i.e. usually less than $5 \%$, is added to the dope solution.

A new formulation of a charged SMM (CSMM) which was anticipated to increase the hydrophilicity of the base polymer (SPEEK) as well as to increase its sulfonic group content was developed. It is well documented that the enhancement of both properties is crucial for a better PEM performance of SPEEK as its proton conductivity is dependent on water uptake and sulfonic group content in its polymer chain [12]. It is expected that the proton conductivity will be further increased by the interaction of the sulfonic group from CSMM with that of SPEEK. Therefore, the objectives of this paper are: to design a novel tailor-made cSMM for blending and modifying SPEEK membranes, to prepare and characterize such nembranes, and to study the said membranes on thermal and mechanical stability.

\subsection{METHODS}

\subsection{Synthesis of Sulfonated Poly (ether ether ketone)}

PEEK was sulfonated following the technique described in $[13,14]$. A 5 g of PEEK was dried in an oven at $100^{\circ} \mathrm{C}$ for $24 \mathrm{hrs}$ and then dissolved in $95 \mathrm{mil}$ of concentrated (95 98\%) sulfuric acid $\left(\mathrm{H}_{2} \mathrm{SO}_{4}\right)$ at room temperature to suppress the heterogeneous sulfonation. After completing the dissolution of PEEK (about $1 \mathrm{~h}$ ), the polymer solution was brought to the desired temperature of $55^{\circ} \mathrm{C}$ and held for 3 hrs to obtain the desired degree of sulfonation (DS). In order to terminate the reaction, the polymer solution was poured into excess ice-cold deionized water under continuous stirring for one night to remove the residual acid. The polymer was washed repeatedly with de-ionized water until a neutral $\mathrm{pH}$ was reached. Then it was dried in air circulation oven at $60^{\circ} \mathrm{C}$ overnight. The formation of SPEEK from

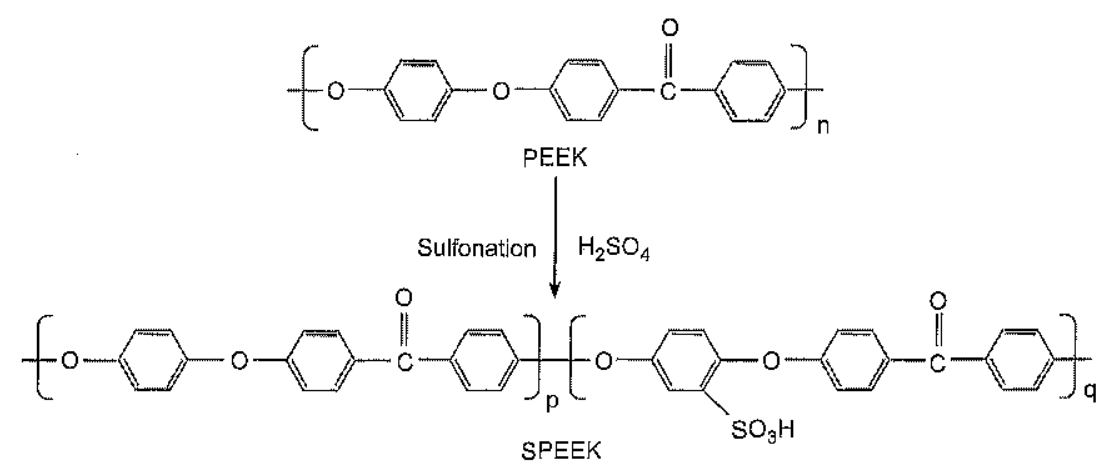

Figure 1 Sulfonation of PEEK 
PEEK is shown in Figure 1. The sulfonated poly (ether ether ketone), SPEEK, is basically a copolymer, consisting of sulfonated PEEK structural unit and original PEEK structural unit.

\subsection{Characterization of Sulfonated Poly(ether ether ketone)}

The characterization of SPEEK was made by measuring the degree of sulfonation (DS) by ${ }^{1} \mathrm{H}$ NMR (Bruker, USA) at a resonance frequency of $400.13 \mathrm{MHz}$ and at room temperature $[14,15]$. The DS is defined as the ratio of the number of the structural units containing sulfonic acid group to the total number of the structural units. The ratio between the peak area of the distinct signal $\left(A H_{f}\right)$ and the integrated peak area of the signals corresponding to all other aromatic hydrogen $\left(A H_{a, b, c, d, e}\right)$ is expressed as

$$
\frac{q}{(12-2 q)}=\frac{A H_{f}}{\sum A H_{a, b, c, d, e}}
$$

Where $q$ is defined as the ratio (the number sulfonated repeat units/the number of the intire repeat units) or the degree of sulfonation (DS\%) (Figure 1). Equation (1) is valid for $0 \leq q \leq 1$.

The ion exchange capacity (IEC) of the membrane was then determined by equation (2) which relates the IEC with DS for SPEEK in hydrogen form.

$$
D S \%=\frac{288(I E C)}{1000-80(I E C)} \times 100
$$

It should be mentioned that the molecular weight of SPEEK in hydrogen form and PEEK are 368 and 288 respectively. The number (80) arises from the difference between these two molecular weights.

\subsection{Synthesis of Charged Surface Modifying Macromolecule}

The charged surface modifying macromolecule, cSMM, end-capped with hydroxyl benzene sulfonate, was synthesized using a two-step solution polymerization method. The initial step involved the reaction of methylene bis (phenyl isocyanate) (MDI) with di (ethylene glycol) (DEG) in a common solvent of dimethylacetamide (DMAc). This mixture formed a urethane prepolymer solution. The prepolymer is a segment-blocked urethane oligomer, poly ( 4,4 'diphenylenemethylene methoxymethylene urethane) having both ends capped with isocyanate. The reaction was then terminated by the addition of Hydroxybenzenesulfonic (HBS) resulting in a solution of charged or sulfonated surface modifying macromolecules (cSMM).

The experimental set-up used for cSMM synthesis is illustrated in Figure 2. The detailed procedures are as follows: to a solution of 0.03 mol $(7.5 \mathrm{~g})$ MDI in $50 \mathrm{~mL}$ of degassed DMAc was loaded in a $1 \mathrm{~L}$ Pyrex round bottom flask. Then, a solution of $0.02 \mathrm{~mol}$ ( $2.122 \mathrm{~g}$ ) degassed DEG in $100 \mathrm{~mL}$ of degassed DMAc was added drop-wise with stirring. The temperature was

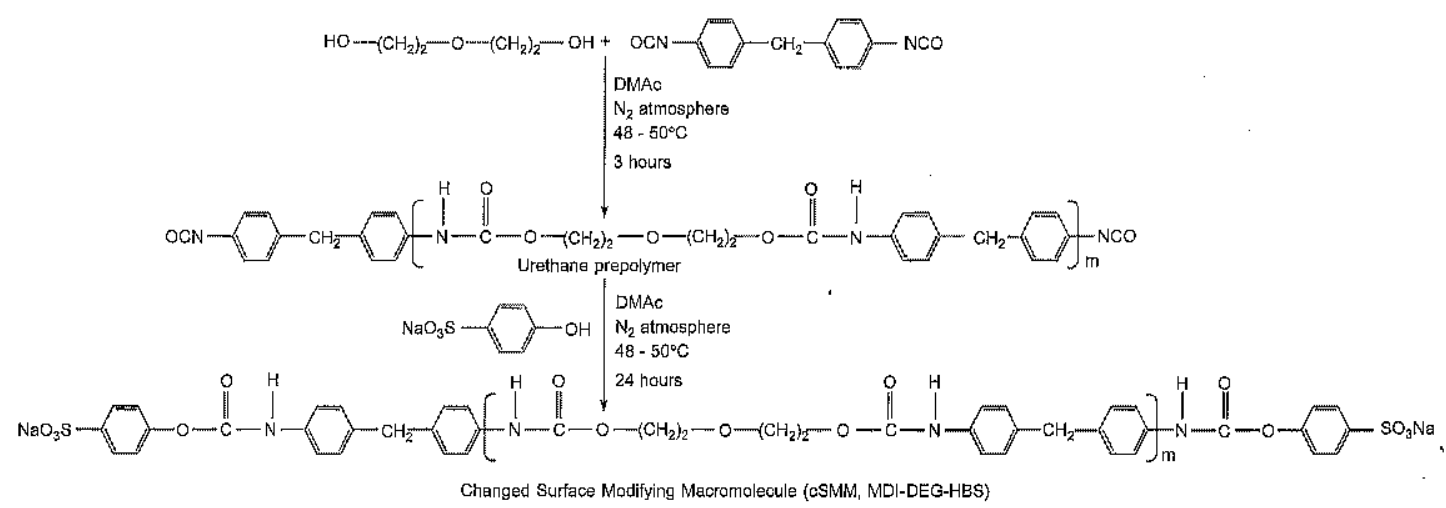

Figure 2 Synthesis of CSMM (MDI-DEG-HBS) 
controlled at $48-50^{\circ} \mathrm{C}$. DEG and MDI were allowed to react for $3 \mathrm{hrs}$. Then $0.02 \mathrm{~mol}(4.644$ g) of HBS dissolved in $50 \mathrm{~mL}$ of degassed DMAc was added drop-wise. The solution was left under stirring for $24 \mathrm{hrs}$ at $48-50^{\circ} \mathrm{C}$, resulting in a solution of charged surface modifying macromolecules. The scheme of the chemical reaction for the synthesis of cSMM (MDI-DEG-HBS) is shown in Figure 2.

\subsection{Characterization of cSMM by FTIR and Elemental Analysis}

The existence of sulfonic group in the CSMM polymer can be confirmed by FTIR Characteristic bands of the aromatic sulfonate salt. The FTIR is also used to confirm the existence of the sulfonic group in the SPEEK membrane as well as the SPEEK/CSMM blend membrane. However, the quantitative analysis of the sulfonic group in cSMM polymer is measured by elemental analysis CHNS analyzer which calculate the sulphur content as weight percent.

\subsection{Preparation of Membranes}

Ten gram of SPEEK was dissolved in $90 \mathrm{~g} 1$ Methyl-2-pyrrolidinone (NMP) to make a $10 \mathrm{wt} \%$ of original SPEEK solution. The blend solutions were prepared by mixing $0.416 \mathrm{~g}$ of each cSMMs and $10 \mathrm{~g}$ of SPEEK, to make $4 \mathrm{wt} \%$ of cSMM in total solid. Both mixtures were stirred for $24 \mathrm{hrs}$, before the mixtures were cast onto a glass plate using a pneumatic casting machine. The membrane was dried at $120^{\circ} \mathrm{C}$ for $24 \mathrm{hrs}$ in a vacuum oven. After cooling to room temperature, the membrane was peeled off from the glass in deionized water. Finally, the membrane was converted into the $\mathrm{H}^{+}$form by immersing it into a $1 \mathrm{M}$ sulfuric acid solution for $24 \mathrm{hrs}$ at the room temperature and blotted dry with absorbent paper before it was air dried.

\subsection{Characterization of Membrane}

\subsubsection{Contact Angle Measurements}

The contact angle, $\mathrm{CA}$, of the membrane surface was measured using a VCA Optima Surface Analysis System (AST Products, Inc., Billerica,
MA). Sample coupons with an area of about $5 \mathrm{~cm} \times 1.5 \mathrm{~cm}$ were prepared by cutting pieces at random locations within the membrane sheet. The sample was placed on a glass plate and fixed with a tape. Then, a drop of distilled water $(2 \mu \mathrm{L})$ was placed on the sample surface using a micro syringe (Hamilton Company, Reno, NV). The CA was measured within a $30 \mathrm{sec}$ period after the water drop was placed. The CAs were measured at ten different spots on each membrane sample coupon and the values are averaged.

\subsubsection{Water Uptake Measurement}

The membrane was dried in an oven at $60^{\circ} \mathrm{C}$ for $48 \mathrm{hrs}$, weighed, soaked in de-ionized water overnight at room temperature, blotted dry with absorbent paper to remove any surface moisture, and re-weighed. Then, $\%$ of water uptake was calculated from the equation below,

$$
\text { Water Uptake } \%=\frac{W_{w e t}-W_{d r y}}{W_{d r y}} \times 100
$$

where $W_{\text {wet }}$ and $W_{d r y}$ are the weight of the wet and the dry membrane respectively. The accuracy of the measurement is within $\pm 7 \%$.

\subsubsection{Fourier Transforms Infrared Spectroscopy}

Fourier transform infrared (FTIR) spectroscopy was used to observe the presence of sulfonic acid groups in CSMM and SPEEK as well as the interactions between SPEEK and CSMM in the blended membrane. The FTIR spectrometer (Magna-IR 560, Nicolet, Madison, MI, USA) equipped with attenuated total reflection (ATR) having a germanium (Ge) spherical crystal. The spectra were measured in absorbance mode over a wave number range of 2000 to $700 \mathrm{~cm}^{-1}$.

\subsubsection{Scanning Electron Microscopy}

The morphology of the blend membrane was investigated using scanning electron microscopy (SEM). Specimen for the SEM was prepared by freezing the dry membrane sample in liquid nitrogen up to $3 \mathrm{~min}$ and breaking it to produce a cross-section. Fresh cross-sectional cryogenic fractures of the membrane was vacuum sputtered with a thin layer of gold by using an ion sputtering 
(Biorad Polaron Division, Hertfordshire, UK) before viewing on the scanning electron microscope (SEMEDAX; XL 40; PW6822/10, Phillips, UK) with a potential of $10 \mathrm{kV}$.

\subsubsection{Thermal Stability Studies}

The glass transition temperature $\left(T_{g}\right)$ was examined by differential scanning calorimeter (DSC) equipped with universal analysis 2000 program (DSC Q1000-0760, TA Instruments, New Castle, DE). Indium was used for the calibration of the temperature. About $10 \mathrm{mg}$ of polymer was crimped into aluminum pan. The polymers were annealed at about $+50^{\circ} \mathrm{C}$ for 10 min under nitrogen atmosphere and then quenched, and scanned at a heating rate of $10^{\circ} \mathrm{C}$ $\min ^{-1}$. The sample weight loss was measured by thermogravimetric analysis (TGA) using thermogravimetric analyzer (Mettler, Toledo, $\mathrm{OH}$ ) . A preoven dried sample of $10 \mathrm{mg}$ was heated under room temperature to $800^{\circ} \mathrm{C}$ at the heating rate $10^{\circ} \mathrm{C} \mathrm{m^{-1 }}$ in air.

\subsubsection{Mechanical Strength Study}

The tensile strength of the membrane was measured using mechanical testing system, MTS (LRX 5 kN, Lloyd Instruments, Fareham, Hants, UK). The gauge length and width of dumbbell tensile specimens were 15 and $4 \mathrm{~mm}$ respectively. The specimen was placed between the grips of the testing machine, and the speed of testing was set at the rate of $5 \mathrm{~mm} \mathrm{~min}{ }^{-1}$. Five specimens were taken from each type of membrane for the measurement and their average value was calculated. The accuracy of the measurement is within $\pm 5 \%$.

\subsection{RESULTS AND DISCUSSION}

\subsection{Polymer Characterization}

Degree of sulfonation of SPEEK was determined using ${ }^{1} \mathrm{H}$ NMR analysis from equation (1) as showi in Figure 3, and was found to be 53.35 DS $\%$. The $H_{f}$ signal is equivalent to the sulfonic acid group content and the other peak signals represent the rest of the hydrogen existing in the SPEEK repeat unit. By using the DS value from ${ }^{1} \mathrm{H}$ NMR analysis, its IEC value was calculated from equation (2) to be $1.613 \mathrm{meq} / \mathrm{g}$.

The existence of sulfonic group in the CSMM polymer can be confirmed by FTIR as shown in Figure 5. Characteristic bands of the aromatic sulfonate salt are observed at 1224, 1069 and $1018 \mathrm{~cm}^{-1}$. The figure proved that the newly
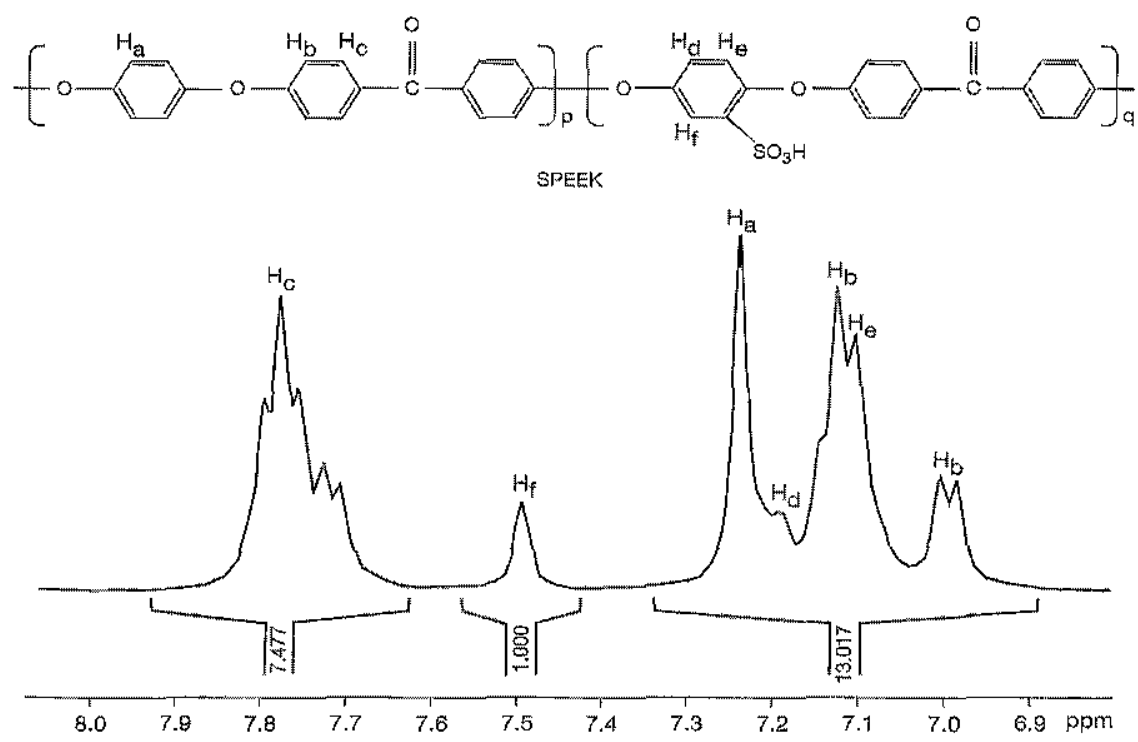

Figure $3 \quad H$ NMR spectrum of SPEEK 
synthesized cSMM contains sulfonic group and its weight percent was calculated by using CHNS elemental analysis. The result reveal that the sulphur content for the cSMM is $1.011 \mathrm{wt} \%$ that can be converted to 0.3441 IEC.

\subsection{Membrane Characterization}

Mixing SPEEK and cSMM in NMP formed homogeneous and transparent solution, however, after casting on a glass plate, the blended membrane was more yellowish than the original SPEEK. Mixing cSMM into SPEEK, due to the similar chemical structure of sulfonic group, are first miscible but then cSMM are expected to migrate upwards to the membrane surface. Therefore, the cSMM has dispersed homogeneously into SPEEK. The homogeneity of dispersions of cSMM in SPEEK was examined with SEM.

Figure 4 show the SEM pictures of high magnification. In both, nodular structures are observed. Judging from the size of each spherical unit, it represents the super nodular aggregates. There is a notable increase in the size of the sphere from the SPEEK to the SPEEK/cSMM blend membrane suggesting an increase in free volume that can be associated with the intake of water molecules.

The SPEEK and SPEEK/cSMM membrane were also analyzed by FTIR. The purpose of FTIR spectroscopy test is to examine the presence of sulfonic group in the membrane samples. The
FTIR spectra of SPEEK and SPEEK/cSMM blend membrane are shown in Figure 5, which is to confirm the presence of sulfonic group in both membranes. The presence of sulfonic group in SPEEK and SPEEK/cSMM blend membrane can be confirmed by the absorption bands at 1222.7 (asymmetric $\mathrm{O}=\mathrm{S}=\mathrm{O}$ ), 1081 (symmetric $\mathrm{O}=\mathrm{S}=\mathrm{O}), 1024(\mathrm{~S}=\mathrm{O})$. FTIR spectra of both membranes were nearly identical. However, by calculating the height of each peak, as presented in Table 1, there is a difference in height at the three particular peaks. This indicates that by adding the CSMM the intensity of the sulfonic group has increased as compared to the original SPEEK.

Table 1 The height of $-\mathrm{SO}_{3} \mathrm{H}$ identity peak in membrane

\begin{tabular}{lccc}
\hline $\begin{array}{l}\text { Peak height } \\
\text { Membrane }\end{array}$ & $\begin{array}{c}\text { Peak } \\
\mathbf{1 2 2 2 . 7}\end{array}$ & $\begin{array}{c}\text { Peak } \\
\mathbf{1 0 8 1}\end{array}$ & $\begin{array}{c}\text { Peak } \\
\mathbf{1 0 2 4}\end{array}$ \\
\hline SPEEK & 0.0566 & 0.0164 & 0.0259 \\
SPEEK/cSMM-DEG & 0.075 & 0.022 & 0.031 \\
\hline
\end{tabular}

The hydrophilicity of the membrane surface can be evaluated by measuring the contact angle (CA). Table 2 shows the results of the CA measurement. SPEEK is significantly higher than SPEEK/cSMM blend at the top surface. The CA
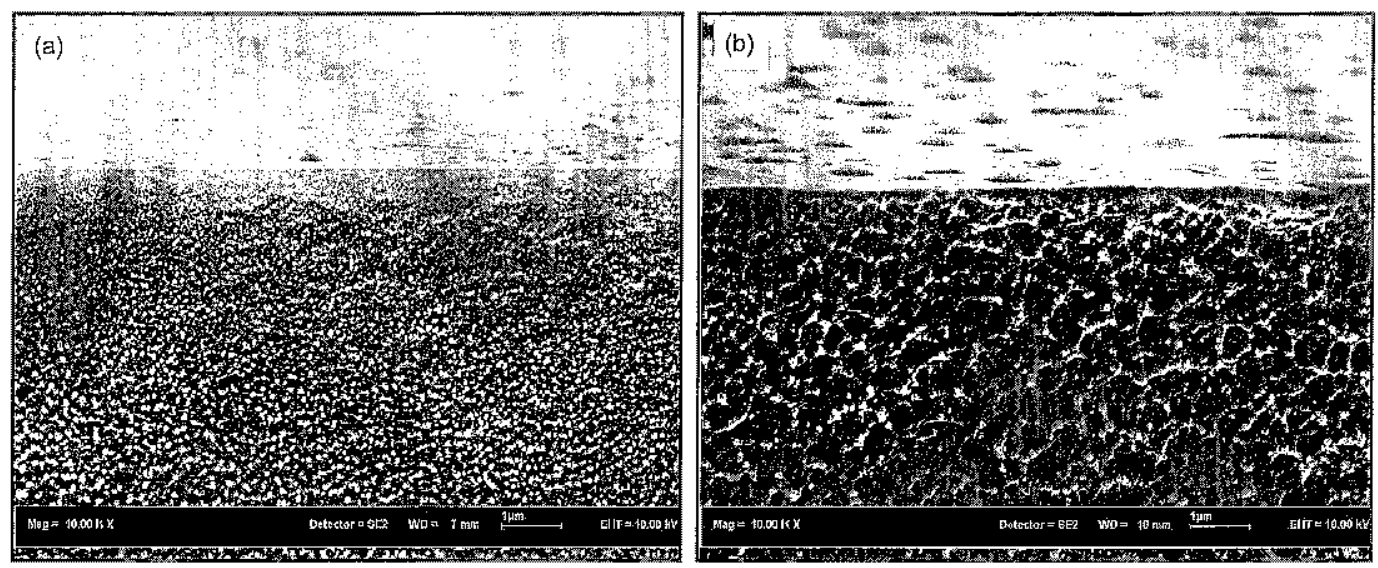

Figure 4 SEM picture of crossectional area of (a) original SPEEK and (b) SPEEK/cSMM 


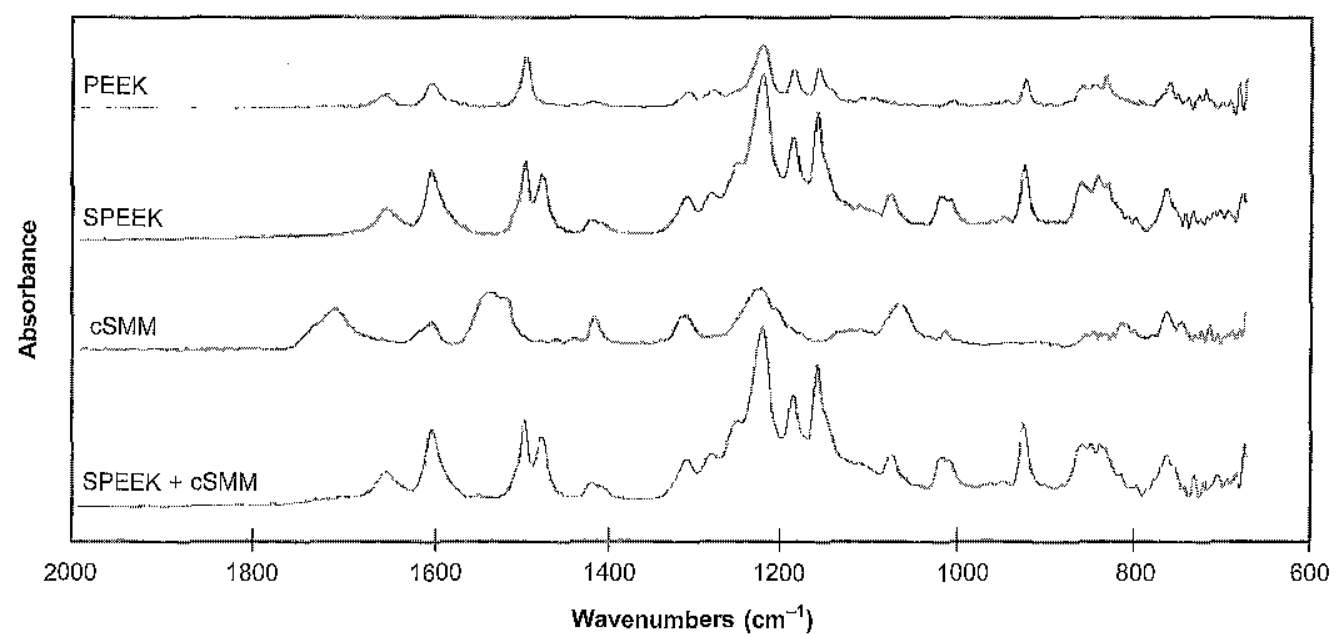

Figure 5 TIR spectra of polymer and membrane in the study

of SPEEK was also higher than SPEEK/cSMM blend at the bottom surface. Comparing the top and the bottom surface, the difference is not significant for SPEEK, while the CA of the top surface was significantly lower than the bottom surface for SPEEK/CSMM blend. This is most likely due to the migration of CSMM to the top surface, covering the surface with the sulfonic acid end groups that are protruded vertical to the surface while the urethane prepolymer part of CSMM is anchored to the host SPEEK.

Table 2 also shows that water uptake increased significantly from SPEEK to SPEEK/cSMM blend. The result shows the total water content of SPEEK is $32 \%$ and the total water content of the blend membrane increased to $38 \%$ due to the increased hydrophilicity and by the additional $\mathrm{SO}_{3} \mathrm{H}$ groups from the cSMM. In SPEEK the sulfonate ions in the ion cluster domain are hydrated with absorbed water molecules [17].
The increase in water uptake means, therefore, the increase in the sulfonate ion density in the cluster [18]. It is likely that the excess sulfonate ions are provided by the added CSMM. In view of the dense structure of the membrane, within a limitation depending on the polarity and acid group content $[18,19]$, the addition of the CSMM hence attracted more water molecules to the strong ionic sites and created the amount of the sorbed water to increase.

\subsection{Thermal Stability}

Thermal stability of Nafion 112, SPEEK, SPEEK/ cSMM blend as well as cSMM was determined by TGA. Figure 6 shows the TGA thermograms. Regarding CSMM, weight decrease of about $5 \%$ was observed as the temperature was raised to $300^{\circ} \mathrm{C}$, followed by a steep decrease in weight of another $75 \%$ when the temperature was raised

Table 2 Characterization of Nafion 112, SPEEK, and SPEEK/cSMM films

\begin{tabular}{cccccc}
\hline Polymer film & $\begin{array}{c}\text { Water } \\
\text { uptake (\%) }\end{array}$ & $\begin{array}{c}\left.\text { CA ( }{ }^{\circ}\right) \\
\text { Top-surface }\end{array}$ & $\begin{array}{c}\left.\text { CA ( }{ }^{\circ}\right) \\
\text { Bottom-surface }\end{array}$ & $\mathbf{T}_{\boldsymbol{g}}\left({ }^{\circ} \mathbf{C}\right)$ & $\begin{array}{c}\text { Tensile } \\
\text { strength } \\
\mathbf{M P a}\end{array}$ \\
\hline Nafion 112 & 20 & 80 & 80 & 143.6 & 34.8 \\
SPEEK & 32 & $83.12 \pm 2.36$ & $84.64 \pm 3.07$ & $151.5,215.4$ & 45 \\
SPEEK/CSMM & 38 & $65.82 \pm 2.97$ & $79.47 \pm 2.80$ & 210.27 & 39 \\
\hline
\end{tabular}




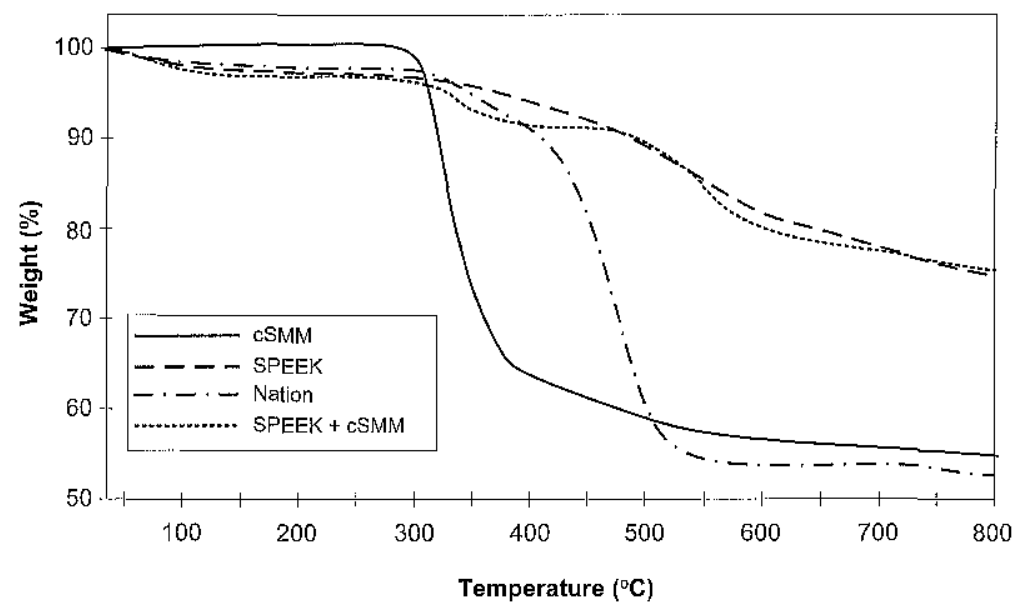

Figure 6 Thermal decomposition curve of polymer and membrane in the study

from $300^{\circ} \mathrm{C}$ to $550^{\circ} \mathrm{C}$. This is due to the removal of sulfonic groups [21] and the decomposition of the polymer chain of polyurethane backbone occurring simultaneously. Compared to cSMM, SPEEK seems far more stable. Since the cSMM is a minor component (4\%) of the polymer blend, its effect on the thermal stability seems only marginal. As for the blend membrane, the decomposition was found to be greater between temperature of $300^{\circ} \mathrm{C}$ to $400^{\circ} \mathrm{C}$ as compared to pristine SPEEK. This is due to the decomposition of sulfonic group in the membrane. This result revealed that the blend membrane contains more sulfonic group than the SPEEK.

The $T_{\mathrm{g}}$ of polymer samples are also shown in Table 2. According to the result, $T_{g}$ for SPEEK were detected at $215.4^{\circ} \mathrm{C}$. The $T_{g}$ of $\mathrm{cSMM}$ and SPEEI/CSMM blend are $114.6^{\circ} \mathrm{C}$ and $210.3^{\circ} \mathrm{C}$, respectively. About $2.4 \%$ reduction in $T_{g}$ of the blend is most probably because of the presence of CSMM has reduced the rigidity of the SPEEK structure.

\subsection{Mechanical Stability}

The results of the tensile strength measurement are presented in Table 2. From the table, the tensile strength of SPEEK is $45 \mathrm{MPa}$ similar to the reported value by [1], while the tensile strength of SPEEK/cSMM blend is $39 \mathrm{MPa}$. Thus, the incorporation of $\mathrm{CSMM}$ in SPEEK has reduced the tensile strength by $14 \%$. This is due to the reduction in the rigidity of the polymer. The tensile strength of SPEEK/CSMM is however still higher than that of Nafion 112.

From the findings, cSMM is clearly a good candidate as an additive for SPEEK in improving its function as polymer electrolyte membrane. The addition of CSMM with an additional $\mathrm{SO}_{3}{ }^{-}$charge has enabled the water uptake to be increased which is crucial to enhance the proton conductivity that of SPEEK.

\subsection{CONCLUSION}

The following conclusions can be drawn from the experimental results.

(1) The newly synthesized cSMM indeed carries charged sulfonic groups.

(2) Surface migration of cSMM occurs when blended into SPEEK membrane.

(3) Blending cSMM into the SPEEK membrane decreases $T_{g}$ and tensile strength.

(4) The SPEEK/cSMM blend membrane absorbs more water as compared to the pristine SPEEK.

(5) CSMM is a potential material to be blended into SPEEK membrane in order to increase its PEM performance. 


\section{ACKNOWLEDGEMENTS}

The author is thankful to the Universiti Telmologi Malaysia for granting him fellowship for his research work at Membrane Research Unit (MRU). The author gratefully acknowledges the financial support from Ministry of Science Technology and Innovation Malaysia for providing financial support through National Science Fellowship scholarship for conducting this research project.

\section{REFERENCES}

[1] Zhong, S., X. Cui, H. Cai, T. Fu, C. Zhao, and H. Na. 2007. Crosslinked Sulfonated Poly(ether ether ketone) Proton Exchange Membranes for Direct Methanol Fuel Cell Applications. J. Power Sources. 164: 65.

[2] Silva, V.S., B. Ruffmann, S. Vetter, A. Mendes, L.M. Mandeira, and S.P. Nunes. 2005. Characterization and Application of Composite Membranes in DMFCs. Catalysis Today. 104: 205-212.

[3] Chang, J.H., J.H. Park, G.G. Park, C.S. Kim, and O.O. Park. 2003. Proton-conducting Composite Membrane Derived from Sulfonated Hydrocarbon and Inorganic Materials. J. Power Sources. 124: 18.

[4] Manea, C., and M. Mulder, 2002. Characterization of Polymer Blends of Polyethersulfone/Sulfonated Polysulfone and Polyethersulfone/Sulfonated Polyetheretherketone for Direct Methanol Fuel Cell Applications. J. Membr. Sci. 20: 443.

[5] Ren, S., C. Li, X. Zhao, Z. Wu, S. Wang, G. Sun, Q. Xin, and X. Yang. 2005. Surface Modification of Sulfonated Poly (ether ether ketone) Membranes using Nafion Solution for Direct Methanol Fuel Cells. J. Membr. Sci. 240: 59.

[6] Choi, W.C., J.D. Kim, and F.I. Woo. 2001. Modification of Proton Conducting Membrane for Reducing Methanol Crossover in a Direct-methanol Fuel Cell. J. Power Sources. 96: 411.

[7] Huang, R.Y.M., P. Shao, C.M. Burns, and X. Feng. 2001. Sulfonation of Poly(ether ether ketone) (PEEK): Kinetic Study and
Characterization. J. Appl. Polym. Sci. 82: 2651.

[8] Suk, D.E., G. Chowdhury, T. Matsuura, R.M. Narbaitz, P. Santerre, G. Pleizier, and Y. Deslandes. 2002. Study on the Kinetics of Surface Migration of Surface Modifying Macromolecules in Membrane Preparation. Macromolecules. 35: 3017.

[9] Rana, D., T. Matsuura, R.M. Narbaitz, and C. Feng. 2005. Development and Characterization of Novel Hydrophilic Surface Modifying Macromolecule for Polymeric Membranes. J. Membr. Sci. 249: 103.

[10] Sulk, D.E., T. Matsuura, H.B. Park, and Y.M. Lee. 2006. Synthesis of a New Type of SurFace Modifying Macromolecules (nSMM) and Characterization and Testing of nSMM Blended Membranes in Membrane Distillation. J. Membr. Sci. 277: 177.

[11] Rana, D., T. Matsurura, and R.M. Narbaitz. 2006. Novel Hydrophilic Surface Modifying Macromolecules for Polymeric Membranes: Polyurethane Ends Capped by Hydroxy Group. J. Membr. Sci. 282: 205.

[12] Li, L., and Y. Wang. 2005. Sulfonated Polyethersulfone Cardo Membranes for Direct Methanol Fuel Cell. J. Membr. Sci. 246: 167.

[13] Jaafar, J., A.F. Ismail, and A. Mustafa. 2007. Physicochemical Study of Poly (ether ether ketone) Electrolyte Membranes Sulfonated with Mixtures of Fuming Sulfuric Acid and Sulfuric Acid for Direct Methanol Fuel Cell Application. Mat. Sci. Eng. A. 460-461: 475.

[14] Zaidi, S.M.J., S.D. Mikhailenko, G.P. Robertson, M.D. Guiver, and S. Kaliaguine. 2000. Proton Conducting Composite Membranes from Poly(Ether Ether Ketone and Heteropolyacids for Fuel Cell Applications. J. Membr. Sci. 173: 17.

[15] Xing, P., G.P. Robertson, M.D. Guiver, S.D. Mikhailenko, K. Wang, and S. Kaliaguine. 2003. Synthesis and Characterization of Sulfonated Poly (ether ether ketone) for Proton Exchange Membranes. J. Membr. Sci. 229: 95.

[16] Kim, D.S., H.B. Park, J.W. Rhim, and Y.M. Lee. 2004. Preparation and Characterization of Crosslinked PVA/ $\mathrm{SiO}_{2}$ Hybrid 